\title{
Task-irrelevant distractors in the delay period interfere selectively with visual short-term memory for spatial locations
}

\author{
Francesco Marini ${ }^{1,2} \cdot$ Jerry Scott $^{1} \cdot$ Adam R. Aron $^{1} \cdot$ Edward F. Ester $^{1,3}$
}

Published online: 24 April 2017

(C) The Psychonomic Society, Inc. 2017

\begin{abstract}
Visual short-term memory (VSTM) enables the representation of information in a readily accessible state. VSTM is typically conceptualized as a form of "active" storage that is resistant to interference or disruption, yet several recent studies have shown that under some circumstances task-irrelevant distractors may indeed disrupt performance. Here, we investigated how task-irrelevant visual distractors affected VSTM by asking whether distractors induce a general loss of remembered information or selectively interfere with memory representations. In a VSTM task, participants recalled the spatial location of a target visual stimulus after a delay in which distractors were presented on $75 \%$ of trials. Notably, the distractor's eccentricity always matched the eccentricity of the target, while in the critical conditions the distractor's angular position was shifted either clockwise or counterclockwise relative to the target. We then computed estimates of recall error for both eccentricity and polar angle. A general interference model would predict an effect of
\end{abstract}

Francesco Marini

francesco.pd@gmail.com

Jerry Scott

jescott@ucsd.edu

Adam R. Aron

adamaron@ucsd.edu

Edward F. Ester

eester@fau.edu

1 Department of Psychology, University of California, San Diego, CA, USA

2 Department of Psychology, University of Nevada, 1664 N Virginia St, Reno, NV 89557, USA

3 Department of Psychology and FAU Brain Institute, Florida Atlantic University, Boca Raton, FL, USA distractors on both polar angle and eccentricity errors, while a selective interference model would predict effects of distractors on angle but not on eccentricity errors. Results showed that for stimulus angle there was an increase in the magnitude and variability of recall errors. However, distractors had no effect on estimates of stimulus eccentricity. Our results suggest that distractors selectively interfere with VSTM for spatial locations.

Keywords Visual working memory · Spatial memory · Short-term memory $\cdot$ Distractor interference

The maintenance of visual information in memory for short periods of time is a ubiquitous demand in everyday life. For example, when we are about to cross a road and look leftwards, we use visual short-term memory (VSTM) to encode information about the location of an oncoming vehicle, and then we maintain this information when we look right. Yet in everyday life we may experience failures of VSTM when task-irrelevant and/or distracting stimuli intervene while maintaining visual information. For example, while holding in mind the location of an oncoming vehicle, we may inadvertently notice the location of a vehicle of the same color parked alongside, and this could affect our existing memory about the location of the first vehicle. How does the disruption occur and which aspects of VSTM are affected?

Several studies have shown that task-irrelevant distractors can disrupt VSTM performance (Berry, Zanto, Rutman, Clapp, \& Gazzaley, 2009; Bettencourt \& Xu, 2016; Clapp \& Gazzaley, 2012; Clapp, Rubens, \& Gazzaley., 2009; Clapp, Rubens, Sabharwal, \& Gazzaley, 2011; Feredoes, Heinen, Weiskopf, Ruff, \& Driver, 2011). However, the mechanisms responsible for this reduction remain a matter of active debate. On the one hand, reductions in memory performance could be 
due to general interference, where a task-irrelevant distractor overwrites or otherwise corrupts the representation of a memory stimulus, resulting in a general decrease in memory performance (Clapp \& Gazzaley, 2012; Clapp et al., 2009; Clapp et al., 2011; Zhang \& Luck, 2009). On the other hand, reductions in memory performance could reflect interactions between attributes of the target and distractor that alter memory representations of the former and lead to reduced memory performance (Magnussen, 2000; Magnussen \& Greenlee, 1992, 1999; Magnussen, Greenlee, Asplund, \& Dyrnes, 1991). In addition to distractor-induced disruptions, VSTM may also be subject to additional sources of general disruption, including temporal decay and interitem interference (Blake, Cepeda, \& Hiris, 1997; Paivio \& Bleasdale, 1974; Ploner, Gaymard, Rivaud, Agid, \& Pierrot-Deseilligny, 1998; White, Sparks, \& Stanford, 1994). However, it has heretofore been unclear whether task-irrelevant distractors cause a similar kind of global interference or whether distractors primarily influence judgments along the feature dimension(s) they share with a remembered stimulus. In a relevant study, Van der Stigchel, Merten, Meeter, and Theeuwes (2007) attempted to discriminate between these alternatives by presenting task-irrelevant distractors while participants remembered the location of a memory probe. Memory performance was biased toward the location of the distractor, but only when distractors were presented in close proximity of the memorized location. This was interpreted as evidence that interference occurred only when the neural representations of target and distractor overlapped in a common spatial map, thus favoring a spatially-specific account of interference over a more general account in which interference would solely depend on the simple presence versus absence of distractors. Here, we extend these findings by examining whether distractors selectively interfere with one of two variables needed to define a point in any two-dimensional coordinate system: polar angle and eccentricity. For example, the visual system computes both polar angle and eccentricity to generate saccade vectors and/or guide covert shifts of attention (e.g., Colby \& Goldberg, 1999; Goldberg \& Bruce, 1990). In the current study, we designed an experimental procedure that allowed us to interfere with the recall of polar angle but not with that of eccentricity and used it to distinguish between general versus selective interference.

We implemented a spatial recall task where participants memorized the location of a target dot and recalled it after a delay that on $75 \%$ of trials included a display of task-irrelevant distractors (see Fig. 1). A previous study (Van der Stigchel et al., 2007) showed that the distance from the target to the recalled position did not differ depending on the presence or absence of distractors, and this argument was used to rule out the global interference account. However, a more sensitive assay of selective (versus global) interference would consist of showing that distractors could selectively interfere with only one (versus both, if global) descriptors of the remembered location - polar angle or eccentricity. Therefore, we systematically changed one dimension (polar angle of the distractor dots relative to the target dot) while fixing the other one (eccentricity) and asked participants to recall the location of the target. On each trial, we measured participants' report errors in terms of polar angle and eccentricity. Notably, the experimental design included one condition with no distractors and two different distractor conditions: a centered distractor condition, in which distractors were shown at the same polar angle as the target, and a shifted distractor condition, in which the distractors were presented at a different polar angle relative to the target. On the one hand, a general interference hypothesis predicts an overall disruption in memory performance that should have a deleterious effect on judgments of both eccentricity and polar angle, irrespectively of the specific distractor condition (i.e., centered or shifted). On the other hand, a selective interference hypothesis predicts that visual information presented during the delay period would merge into an existing memory representation to modulate subsequent recall. Therefore, because distractors were presented always at the same eccentricity and at a different polar angle relative to the target, the selective interference account predicts that distractors would influence polar angle judgments but not eccentricity judgments specifically in the shifted distractor condition (relative to the centered and no distractor conditions). Additionally, we conducted curve fitting analyses in order to investigate whether any observed memory interference effect was due to an attraction effect similar to the one previously reported (Van der Stigchel et al., 2007) or to changes in the precision of the memory representation (or both).

\section{Experiment 1}

\section{Method}

\section{Participants}

Eighteen UC San Diego undergraduate volunteers participated for course credit (mean age $\pm S D: 20.1 \pm 1.4$, eight males, 17 right-handed). All participants had normal or corrected-tonormal vision, no history of neurological impairments, and gave both written and oral informed consent as required by the local Institutional Review Board.

\section{Stimuli}

The experiment was run on an iMac (21.5-inch monitor, 1920 $\times 1080$ pixels, $60 \mathrm{~Hz}$, gamma corrected) using custom-built code in MATLAB R2015a (MathWorks, Inc.) and Psychtoolbox 3.0.12 (Kleiner et al., 2007). Participants were seated $63 \mathrm{~cm}$ from the monitor (eyes to screen) and used a chin 


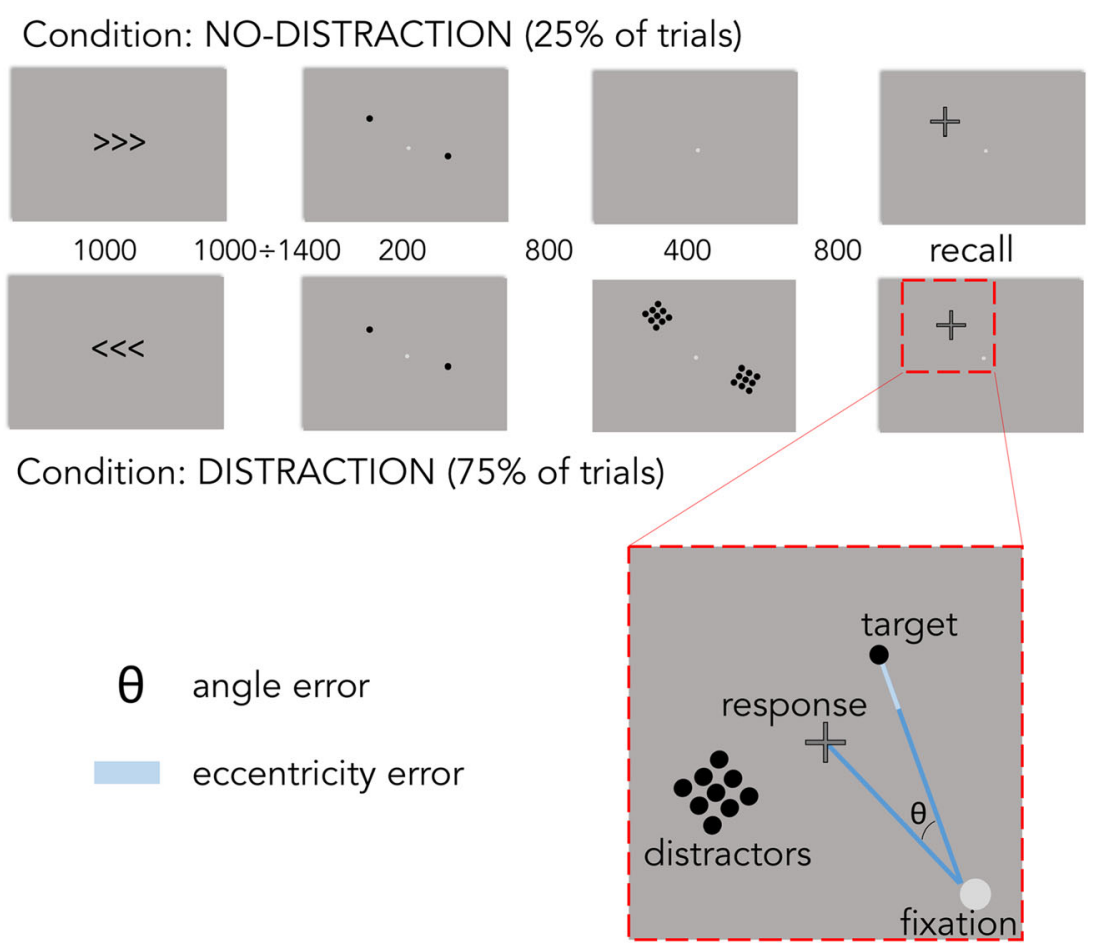

Fig. 1 Experimental design. a Each experiment included 25\% no distractor trials and $75 \%$ distractor trials. Stimuli are enlarged for illustrative purposes. Numbers represent the duration of each screen display in ms. Cross represents the response cursor. b Enlarged area at the bottom right shows relative locations of target, distractor, and participant's response on a single trial. This illustrates how the two

dependent measures (angle error, eccentricity error) used in this study were computed. The magnitude of the polar angle theta (in degrees) corresponds to the angle error, and the length of the light blue line (in degrees of visual angle) corresponds to the eccentricity error. (Color figure online)

rest. Each trial involved the sequential presentation of several visual displays on a dark-gray background (see Fig. 1a): a fixation display for 1,000-1,400 ms (jittered intertrial interval, fixation dot width: $0.1^{\circ}$ of visual angle), a cue display for $500 \mathrm{~ms}$ (leftward or rightward arrows), a stimulus display for $200 \mathrm{~ms}$ (one light-gray dot in each hemifield, dot width in degrees of visual angle: $0.18^{\circ}$, dot eccentricity: $3^{\circ}-4^{\circ}$, jittered across trials but fixed within trial), a fixation display for 2,000 $\mathrm{ms}$ (intermixed with a distractor display displayed $800 \mathrm{~ms}$ after stimulus offset for a duration of $400 \mathrm{~ms}$ on $75 \%$ of trials), and a fixation/response display (presented until a response occurred). Targets were presented along the periphery of an imaginary circle centered at fixation with a radius between 3 and 4 degrees of visual angle (randomly chosen on each trial from a uniform distribution and always matching the eccentricity of the center of the distractor cluster presented on the same trial). The nontarget stimulus, which was displayed in the contralateral hemifield relative to the target during the stimulus display phase, had the same eccentricity as the target and a randomly selected polar angle. When present, the distractor display included two clusters of nine dots each (light gray, one cluster per hemifield, dot width in degrees of visual angle: $0.18^{\circ}$, cluster width: $1.2^{\circ}$ ). Unbeknownst to participants, distractor clusters were located at the same eccentricity and either the same $(25 \%$ of trials, centered distractor

condition) or at a different polar angle of the target dot (50\% of trials, shifted distractor condition; angular offset $12^{\circ}-18^{\circ}$ randomly chosen on each trial from a uniform distribution, always counterclockwise). The remaining $25 \%$ of trials had no distractor display (no distractor condition). The response display contained a cross-shaped cursor (width: $0.7^{\circ}$ of visual angle) that participants controlled using a mouse.

\section{Procedure}

Participants received both written and oral instructions. Participants had to fixate the screen center and to direct their attention covertly to the visual hemifield cued by the arrow. They were instructed to remember the spatial location of the dot presented in the relevant hemifield (hereafter referred to as the target) and recall this location at the end of the trial. Participants were instructed that distractors could appear but were task-irrelevant and should be ignored. The experiment included 16 practice trials followed by 384 trials divided in 12 blocks (the entire experiment had a duration of about 1 hour). On each trial, we computed eccentricity and polar angle error estimates by computing the absolute value of eccentricity and angular differences, respectively, between the target location and the participants' response (see Fig. 1b). Trials where angular estimates were larger than twice the polar angle 
separating target and distractors and trials where eccentricity estimates were greater or less than half of the target eccentricity were deemed as outliers and eliminated (approximately $4.6 \%$ of total trials).

Polar angle and eccentricity recall errors were compared across conditions using repeated-measures analyses of variance (ANOVAs). Each ANOVA included distraction type as a threelevel factor: no distractor, centered distractor, and shifted distractor. Where appropriate, $p$ values were corrected for nonsphericity using the Greenhouse-Geisser procedure. Post hoc comparisons, when appropriate, were conducted using paired $t$ tests corrected for multiple comparisons with the Holm-Bonferroni method (Holm, 1979). Effect sizes are reported as partial eta squared $\left(\eta_{\mathrm{p}}{ }^{2}\right)$ values for factorial analyses (ANOVAs) and Cohen's $d_{z}$ (Cohen, 1988) for paired $t$ tests.

In order to quantify changes in recall estimates and overall precision, in a separate analysis individual distributions of signed polar angle recall errors were fitted with a von Mises distribution (circular Gaussian), which describes the data in terms of central tendency $(\mathrm{mu})$ and variability $(\mathrm{k})$ and thus adds a variability parameter with respect to the standard ANOVA on individual means. Goodness of fit was evaluated by calculating the $R^{2}$ index. Across-subject averages of the von Mises parameters were analyzed using an ANOVA, with distraction type as a three-level factor (no distractor, centered distractor, shifted-counterclockwise distractor). Data were analyzed using MATLAB R2015a (MathWorks, Inc.), SPSS Statistics (IBM Corp.) and G-Power 3.1 (Faul, Erdfelder, Lang, \& Buchner, 2007).

\section{Results}

The analysis of polar angle recall errors identified a main effect of distraction type, $F(2,16)=4.540, p=.032, \eta_{\mathrm{p}}{ }^{2}=$ 0.211 , such that errors were larger on shifted distractor trials relative to no distractor trials (mean polar angle error $\pm 1 S E$ : $5.25^{\circ} \pm 0.28^{\circ}$ and $4.79^{\circ} \pm 0.24^{\circ}$, respectively) $t(17)=4.196, p$ $=.001$, corrected $\alpha=.017, d_{z}=0.989$ (see Fig. 2a). The difference between Centered and Shifted Distractor trials was not significant after correction for multiple comparisons, $t(17)=2.119, p=.049$, corrected $\alpha=.025, d_{z}=0.499$. No significant difference was observed between centered and no distractor trials, $t(17)=0.022, p=.983$, corrected $\alpha=.05$.

The analysis of eccentricity errors also identified a main effect of distraction type, $F(2,16)=4.156, p=.024, \eta_{\mathrm{p}}{ }^{2}=$ 0.196 , with a significant reduction in eccentricity errors for centered versus no distractor trials (mean eccentricity error \pm 1 $S E: 0.376 \pm 0.023$ and $0.411 \pm 0.026$, respectively, in degrees of visual angle) $t(17)=2.721, p=.015$, corrected $\alpha=.017, d_{z}=$ 0.641 (see Fig. 2b). No statistically-significant difference was observed on shifted distractor trials relative to centered and no distractor trials, respectively, $t(17)=2.013, p=.06$, corrected $\alpha$ $=.025$, and $t(17)=1.127, p=.27$, corrected $\alpha=.05$.
A

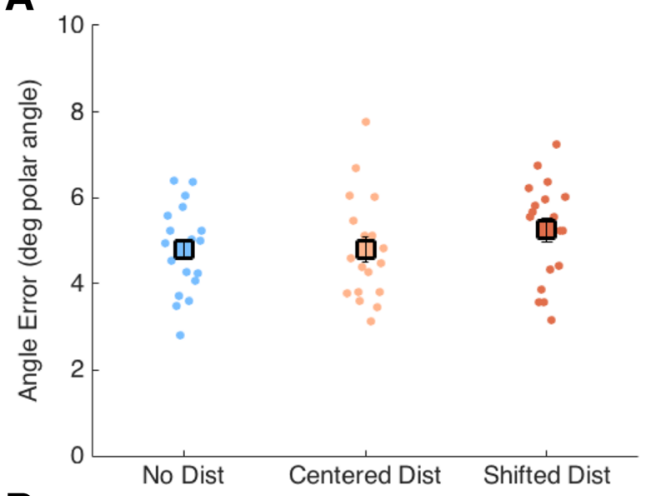

B
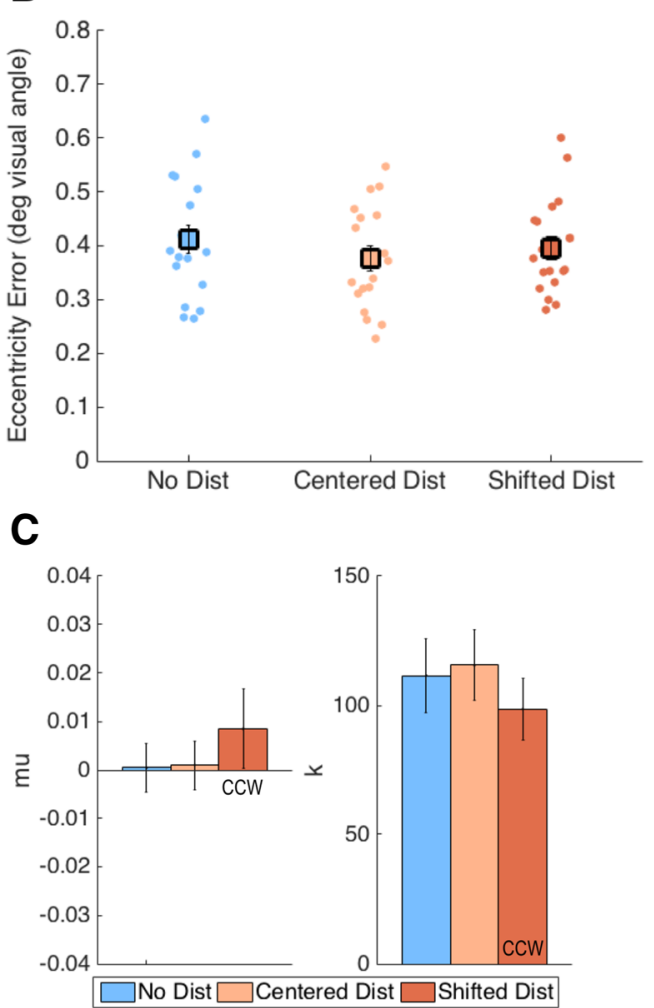

Fig. 2 Results of Experiment 1. a Individual (circles) and group mean (square symbol) of the absolute polar angle recall error across the three experimental conditions. Error bars are \pm 1 standard error of the mean (SEM). b Individual (circles) and group mean (square symbol; error bars are $\pm 1 S E M$ ) of eccentricity error for the angular position of the target across the three experimental conditions. $\mathbf{c}$ Mean values of the parameter estimates of the von Mises distributions ( $m u$ : left, $k$ : right). Positive values of the central tendency parameter mu correspond to counterclockwise errors in the position of the response relative to the target; negative values represent clockwise errors. Note that in the shifted distractor condition distractors were always shifted counterclockwise relative to the target $(C C W)$. Error bars are $\pm 1 S E M$. (Color figure online)

Increases in polar angle errors could be caused by systematic recall errors (either toward or away from the distractor), an overall increase in report variability, or a mixture of both. Therefore, we quantified magnitude and variability of angular errors by fitting each participant's data with a von Mises distribution (the 
circular analog of a standard Gaussian) with mean $m u$ and concentration (the reciprocal of dispersion) $k$. This model provided a good description of subjects' performance; the average $( \pm 1$ $S E M) R^{2}$ values for fits to error distributions in the no distractor, centered distractor, and shifted distractor conditions were 0.89 $( \pm 0.02), 0.90( \pm 0.02)$, and $0.93( \pm 0.01)$, respectively. Average estimates of $m u$ and $k$ were entered into separate ANOVAs with distraction type (no distractor, centered distractor, shiftedcounterclockwise distractor) as the sole within-subject factor. No significant effect of distraction type was found on the $m u$ parameter, $\mathrm{F}(2,16)=1.005, p=.355$, or on the $k$ parameter, $F(2$, 16) $=2.237, p=.144$.

\section{Discussion}

Experiment 1 showed that VSTM for spatial location is influenced by irrelevant delay-period distractors, as indicated by both increased polar angle recall errors (non-matched between stimulus and distractors) and reduced eccentricity recall errors (matched between stimulus and distractors). Recall errors on Shifted Distractor trials were significantly larger relative to No Distractor trials yet not significantly when compared to Centered Distractor trials. Possibly, our sample did not achieve the necessary statistical power to identify a smaller effect relative to that found for the Shifted vs. No Distractor comparison (i.e., $\mathrm{d}_{\mathrm{z}}=0.50$ and $\mathrm{d}_{\mathrm{z}}=0.90$, respectively). However, why the magnitude of the angular error effect was smaller when Shifted Distractor trials were compared to Centered versus No Distractor trials remains an open question. Some methodological aspects of this Experiment may limit the generality of the results: (i) in the Centered Distractor condition, distractor clusters always appeared at target's location with no spatial jitter, therefore it cannot be excluded that some participants may have used it to cue recall; (ii) in the Shifted Distractor condition, distractor clusters were always rotated in the counter-clockwise direction, rather than counterbalanced (50\% of trials clockwise and 50\% counter-clockwise), and this might have caused participants to implement response strategies (e.g. always click in a clockwise position with respect to the distractor). Therefore, Experiment 2 was conducted in order to replicate the findings of Experiment 1 while making appropriate adjustments to address these issues.

\section{Experiment 2}

\section{Method}

\section{Participants}

Eighteen volunteers participated in the study for course credit or payment (mean age $\pm S D: 23.1 \pm 1.4$, eight males, all selfreportedly right-handed). All participants had normal or corrected-to-normal vision, no history of neurological impairments, and all gave both written and oral informed consent as required by the local Institutional Review Board.

\section{Stimuli}

The experiment was run on a Mac mini (20.1-inches Dell 2007FP monitor, $1024 \times 768$ pixels, $60 \mathrm{~Hz}$, gamma corrected) using custom-built code in MATLAB R2015a (MathWorks, Inc.) and Psychtoolbox 3.0.12 (Kleiner et al., 2007). Participants were seated $57 \mathrm{~cm}$ from the monitor (eyes to screen) and used a chin rest. Each trial involved the presentation of several visual displays with the same characteristics and timing of Experiment 1 (see Fig. 1a) except for minor changes as to the size of visual stimuli (memory stimuli dot width: $0.3^{\circ}$, dot eccentricity: $3.5^{\circ}-4.5^{\circ}$; distractor stimuli dot width: $0.25^{\circ}$, cluster width: $1.2^{\circ}$; all measures in degrees of visual angle). Two other aspects differed from Experiment 1: (i) in all distractor conditions (including centered distractor), the distractor cluster was spatially jittered of $\pm 3^{\circ}$ of polar angle, and (ii) in the shifted distractor condition, the direction of rotation of the distractor clusters was counterbalanced $(50 \%$ of trials clockwise and $50 \%$ counterclockwise).

\section{Procedure}

The experiment and analysis procedure were identical to those described for Experiment 1. Because in the shifted distractor condition of Experiment 2 distractors were shifted either clockwise or counterclockwise (angular offset $12^{\circ}-18^{\circ}$ randomly chosen on each trial from a uniform distribution), the von Mises fitting was conducted separately for each direction. Accordingly, the ANOVA on von Mises parameters factored distraction type as a four-level factor (no distractor, centered distractor, shifted distractor clockwise, and shifted distractor counterclockwise).

\section{Results}

For the analysis of recall errors, two one-way repeated-measures analyses of variance (ANOVAs) were implemented, as described for Experiment 1. These ANOVAs used as dependent measures the absolute recall errors in polar angle and in eccentricity, respectively. Each ANOVA included distraction type as a three-level factor (no distractor, centered distractor, shifted distractor).

The angle error ANOVA identified a main effect of distraction type, $F(2,16)=11.181, p=.001, \eta_{\mathrm{p}}{ }^{2}=0.397$, such that polar angle recall errors were significantly larger on shifted distractor relative to no distractor trials, $t(17)=5.824, p<$ .001 , corrected $\alpha=.017, d_{z}=1.373$, but not relative to centered distractor trials, $t(17)=2.328, p=.032$, corrected $\alpha=$ $.025, d_{z}=0.549$. No significant difference was observed 
between centered and no distractor trials, $t(17)=1.979, p=$ .064 , corrected $\alpha=.05$ (mean polar angle error $\pm 1 S E$ : shifted distractor $5.05^{\circ} \pm 0.26^{\circ}$, centered distractor $4.43^{\circ} \pm 0.24$, and no distractor $4.06^{\circ} \pm 0.16^{\circ}$; see Fig. $3 \mathrm{a}$ ). The analysis of eccentricity recall errors revealed no effect of distraction type, $F(2,16)=.218, p=.806$ (see Fig. 3b). This may be because, unlike Experiment 1, here distractor clusters were spatially jittered and therefore they could not be used to cue recall.

As before, we examined recall errors or changes in the variability of polar angle estimates across conditions by fitting each participant's data with a von Mises distribution. The average $R^{2}$ values and their standard errors were as follows: no distractor $0.90 \pm 0.01$, centered distractor $0.91 \pm 0.01$, shiftedclockwise distractor, $0.91 \pm 0.01$, and shifted-counterclockwise distractor $0.90 \pm 0.02$. Average estimates of $m u$ were compared across conditions using ANOVAs, which did not reveal any significant effect, $F(3,51)=1.072, p=.324$. The ANOVA on the $k$ parameter showed a main effect of distraction type, $F(3,51)=4.909, p=.015, \eta_{\mathrm{p}}^{2}=0.224$. Parameter estimates were significantly smaller - thus indicating increased variability of the distribution - in the shifted distractor conditions (both counterclockwise and clockwise) compared to the no distractor condition, $t(17)=5.239, p<.001$, corrected $\alpha=.008, d_{z}=$ 1.235 , and $t(17)=3.473, p<.005$, corrected $\alpha=.01, d_{z}=$ 0.819 , for counterclockwise and clockwise distractors, respectively. The remaining four post-hoc comparisons did not yield statistically significant results (all $p \mathrm{~s}>.07$ ).

\section{Discussion}

The results of Experiment 2 were generally consistent with the results of Experiment 1. In both experiments, we observed larger polar angle errors on shifted distractor relative to no distractor trials (although the effect was much smaller, and statistically nonsignificant, when shifted distractors trials were compared to centered distractor trials). Experiment 2 clarified that this effect likely reflects an increase in response variability during shifted distractor relative to no distractor trials. Conversely, centered distractors were associated with smaller eccentricity errors in Experiment 1 but had no effect in Experiment 2. Thus, the preponderance of evidence suggests that distractors disrupted memory for polar angle while having minimal effect on memory for eccentricity. This pattern is consistent with a selective interference account where distractors interfere with memory representations along a task-relevant dimension.

\section{General discussion}

Many previous VSTM studies reported that delay-period distractors have deleterious effects on memory performance (Berry et al., 2009; Clapp \& Gazzaley, 2012; Clapp et al.,

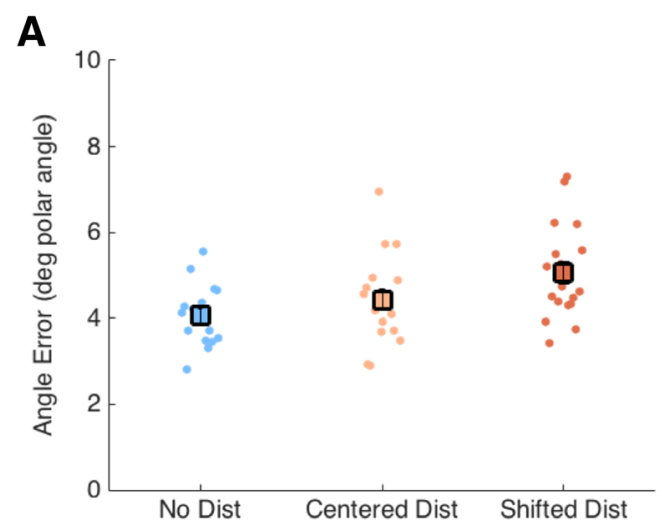

B

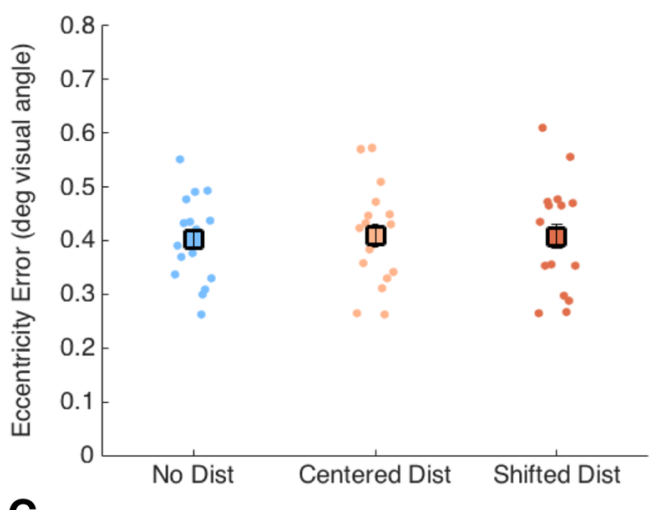

C

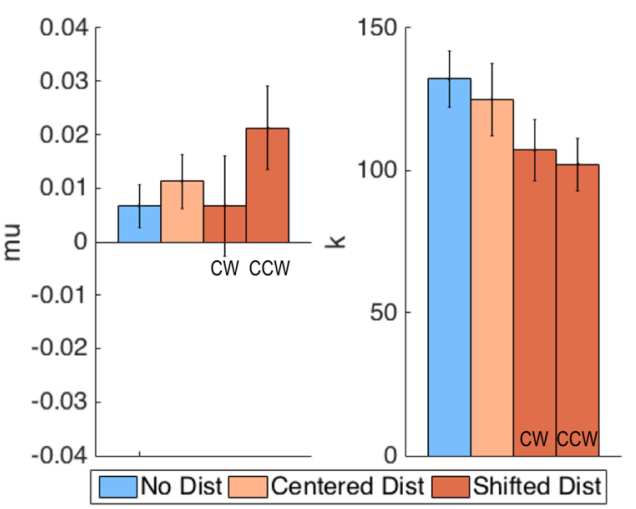

Fig. 3 Results of Experiment 2. a Individual (circles) and group mean (square symbol) of recall error for the angular position of the memorized stimulus across the three experimental conditions. Error bars are \pm 1 standard error of the mean (SEM). b Individual (circles) and group mean (square symbol; error bars are $\pm 1 S E M$ ) of eccentricity error for the angular position of the memorized stimulus across the three experimental conditions. c Mean values of the parameter estimates of the von Mises distributions ( $m u$ : left, $k$ : right). Positive values of the central tendency parameter mu correspond to counterclockwise errors in the position of the response relative to the target; negative values represent clockwise errors. Note that in the shifted distractor condition distractors were shifted either clockwise $(C W)$ or counterclockwise $(C C W)$. Error bars are \pm 1 SEM. (Color figure online)

2009; Clapp et al., 2011; Feredoes et al., 2011), but the sources of these effects are unclear. On the one hand, decreased memory performance could reflect a general disruption in memory 
representations. On the other hand, deleterious effect on memory caused by distractors may be more selective and limited to a single critical dimension (in the case of our study, polar angle). Distinguishing between these accounts was the objective of this study.

Here, we developed an experimental design that allowed us to separate eccentricity and polar angle errors in spatial recall in order to tease apart selective from general interference effects. The general interference account predicts that distractors should increase recall error for both polar angle and eccentricity, while the selective interference account predicts that distractors should increase recall error for polar angle but not eccentricity. Our results are consistent with the latter alternative. In Experiment 1, distractor-related modulations were twofold, consisting of both larger recall errors for polar angle, on which the memory target and the distractor did not match, and reduced recall errors for eccentricity, on which the target and the distractor always matched. In Experiment 2, we replicated the effect of distractors on polar angle recall errors but found no effect of distractors on estimates of eccentricity. Moreover, model fitting in Experiment 2 revealed that the larger polar angle errors in shifted distractor conditions were associated with a significant increase in the variability of the recalled location. Together these studies show that irrelevant information presented during delay interferes with VSTM for spatial location through a selective interference mechanism (i.e., recall error interfered with judgments of polar angle and either improved or had no effect on judgments of eccentricity).

It is known that the magnitude of interference effects driven by delay-period distractors is particularly large when distractors differ from the memory-relevant stimulus feature along a task-relevant dimension (e.g., spatial frequency or orientation; Magnussen et al., 1991; Magnussen \& Greenlee, 1992). These results have been interpreted as evidence that VSTM representations are stored is feature-specific channels in midlevel visual cortex and interference effects manifest when channels overlap (Magnussen, 2000; Magnussen \& Greenlee, 1999). Motivated by this work, the current study manipulated the polar angle separating a distractor from a to-be-remembered target while presenting both targets and distractors at the same eccentricity. Interference effects manifested specifically on polar angle but not on eccentricity. This outcome is inconsistent with a general interference account of VSTM interference and rather provides support for the selective interference account. However, the interference effects observed here - despite being associated with large statistical effect-size values - appear to be of small absolute magnitudes, and therefore the extent to which spatial distractors are capable to shift existing VSTM representations for spatial location is probably limited.

It is worth observing that the reduction of eccentricity error observed in Experiment 1 is compatible with the possibility that participants used distractor information on centered distractor trials as a cue to guide recall. However, if eccentricity was used systematically as a recall cue, then eccentricity errors should have been larger also on no distractor trials relative to shifted distractor trials, which was not the case. Moreover, the similar result was absent from Experiment 2, where no differences in eccentricity errors were observed depending on distractor condition. If distractors were used as recall cues, larger eccentricity recall errors should have manifested on no distractor trials relative to both centered and shifted distractor trials, but this was not observed. Therefore, it appears unlikely that participants systematically used eccentricity information from the distractors as cues to guide their spatial recall estimates. Another possibility is that participants learned the eccentricity of the stimuli, which was maintained within one degree throughout the experiment. Ideally, in a potential follow-up experiment, eccentricity could be varied within a broader range, in order to prevent possible participants' learning.

The current findings are reminiscent of an earlier study (Van der Stigchel et al., 2007), which investigated the effects of irrelevant visual onsets on VSTM for spatial location, and extend it in several important ways. Our results accord with the general findings reported by Van der Stigchel's et al. (2007) that spatial memory representations are modulated by irrelevant visual stimuli presented during the delay period while revealing several new insights. First, because the similarity of features between target and distractor elements appears to be crucial for VSTM interference effects (Magnussen \& Greenlee, 1992; Magnussen et al., 1991) we used equally sized dots both as memory targets (constituted by a single dot presented in isolation) and as distractors (constituted by clusters of dots), while Van der Stigchel's study used crosses and circles, respectively. Moreover, our approach controls stimulus eccentricity within each trial while fractionating recall into two different performance measures (angle and eccentricity). This allowed us not only to match the eccentricity of memory stimuli and distractors at the single-trial level, which was not done in the previous study, but also to show that distractor interference shapes memory for polar angle and not for eccentricity, thus providing more direct support for the selective interference account. In addition, we conducted curve-fitting analyses that allowed us to identify that the parameter compromised by the presence of distractors is the variability of the memory representation. This is reminiscent of earlier work that showed how disruptions of working memory representation occurring at long delay intervals typically manifest as increases in the variability of the recall errors, possibly due to build-up of internal noise in the memory system (Blake et al., 1997; Paivio \& Bleasdale, 1974; Ploner et al., 1998; White et al., 1994). In contrast, Van der Stigchel and colleagues (2007) found that location reports were shifted in the direction of the irrelevant visual onset stimulus, thus 
suggesting spatial attraction of the memory representation toward the location of the distractor. Although we did not find significant evidence of spatial attraction, the scope of our study was different because we focused on the characterization of the interference mechanism in terms of global or selective.

Apart from reports of detriments in memory accuracy when interfering stimuli were presented during maintenance, only a few studies so far have provided direct support for selective interference in VSTM by irrelevant sensory information presented during maintenance (Dubé, Zhou, Kahana, \& Sekuler, 2014; Huang \& Sekuler, 2010; Magnussen \& Greenlee, 1999; Rademaker, Bloem, De Weerd, \& Sack, 2015). These memory interference effects have been documented more often for features such as orientation or spatial frequency and only once for spatial location (Van der Stigchel et al., 2007), which is thought to rely on different neural mechanism relative to VSTM for object features (Courtney, Ungerleider, Keil, \& Haxby, 1996; Smith et al., 1995). The latter is a natural choice, as locations can be characterized via a combination of many variables including eccentricity and polar angle. We reasoned that global interference would have caused a disruption in both eccentricity and polar angle estimates, while selective interference would have caused a disruption of polar angle only (given that our design manipulated the angular distance between target and distractors while keeping the same eccentricity). Because we found that the angular precision of the recall deteriorated (Experiment 1 and 2) while the eccentricity precision improved (Experiment 1) or was unaffected (Experiment 2), we conclude that the information content of distracting stimuli penetrates VSTM by producing a selective interference consisting of dimension-specific modulations of the relevant memory representation. Thus, the present study contributes to advance the understanding of the mechanisms of delay-period interference in VSTM for spatial location.

Acknowledgements We acknowledge funding from a James S. McDonnell Scholar Award 220020375 to A.R.A.

\section{Compliance with ethical standards}

Conflict of interest The authors declare no competing financial interests.

\section{References}

Berry, A. S., Zanto, T. P., Rutman, A. M., Clapp, W. C., \& Gazzaley, A. (2009). Practice-related improvement in working memory is modulated by changes in processing external interference. Journal of Neurophysiology, 102(3), 1779-1789.

Bettencourt, K. C., \& Xu, Y. (2016). Decoding the content of visual shortterm memory under distraction in occipital and parietal areas. Nature Neuroscience, 19(1), 150-157.
Blake, R., Cepeda, N. J., \& Hiris, E. (1997). Memory for visual motion. Journal of Experimental Psychology: Human Perception and Performance, 23(2), 353.

Clapp, W. C., \& Gazzaley, A. (2012). Distinct mechanisms for the impact of distraction and interruption on working memory in aging. Neurobiology of Aging, 33(1), 134-148.

Clapp, W. C., Rubens, M. T., Sabharwal, J., \& Gazzaley, A. (2011). Deficit in switching between functional brain networks underlies the impact of multitasking on working memory in older adults. Proceedings of the National Academy of Sciences, 108(17), 72127217.

Clapp, W. C., Rubens, M. T., \& Gazzaley, A. (2009). Mechanisms of working memory disruption by external interference. Cerebral Cortex, 20(4), 859-872.

Cohen, J. (1988). Statistical power analysis for the behavioral sciences (2nd ed.). Hillsdale: Erlbaum.

Colby, C. L., \& Goldberg, M. E. (1999). Space and attention in parietal cortex. Annual Review of Neuroscience, 22, 319-349.

Courtney, S. M., Ungerleider, L. G., Keil, K., \& Haxby, J. V. (1996). Object and spatial visual working memory activate separate neural systems in human cortex. Cerebral Cortex, 6(1), 39-49.

Dubé, C., Zhou, F., Kahana, M. J., \& Sekuler, R. (2014). Similarity-based distortion of visual short-term memory is due to perceptual averaging. Vision Research, 96, 8-16.

Faul, F., Erdfelder, E., Lang, A. G., \& Buchner, A. (2007). G*Power 3: A flexible statistical power analysis program for the social, behavioral, and biomedical sciences. Behavior Research Methods, 39(2), 175191.

Feredoes, E., Heinen, K., Weiskopf, N., Ruff, C., \& Driver, J. (2011). Causal evidence for frontal involvement in memory target maintenance by posterior brain areas during distracter interference of visual working memory. Proceedings of the National Academy of Sciences, 108(42), 17510-17515.

Goldberg, M. E., \& Bruce, C. J. (1990). Primate frontal eye fields III: Maintenance of a spatially accurate saccade signal. Journal of Neurophysiology, 64, 489-508.

Holm, S. (1979). A simple sequentially rejective multiple test procedure. Scandinavian Journal of Statistics, 6(2), 65-70.

Huang, J., \& Sekuler, R. (2010). Distortions in recall from visual memory: Two classes of attractors at work. Journal of Vision, 10(2), 24 24.

Kleiner, M., Brainard, D., Pelli, D., Ingling, A., Murray, R., \& Broussard, C. (2007). What's new in Psychtoolbox-3. Perception, 36(14), 1.

Magnussen, S. (2000). Low-level memory processes in vision. Trends in Neurosciences, 23(6), 247-251.

Magnussen, S., \& Greenlee, M. W. (1992). Retention and disruption of motion information in visual short-term memory. Journal of Experimental Psychology: Learning, Memory, and Cognition, 18, 151.

Magnussen, S., \& Greenlee, M. W. (1999). The psychophysics of perceptual memory. Psychological Research, 62(2/3), 81-92.

Magnussen, S., Greenlee, M. W., Asplund, R., \& Dyrnes, S. (1991). Stimulus-specific mechanisms of visual short-term memory. Vision Research, 31(7), 1213-1219.

Paivio, A., \& Bleasdale, F. (1974). Visual short-term memory: A methodological caveat. Canadian Journal of Experimental Psychology, 28,24 .

Ploner, C. J., Gaymard, B., Rivaud, S., Agid, Y., \& Pierrot-Deseilligny, C. (1998). Temporal limits of spatial working memory in humans. European Journal of Neuroscience, 10(2), 794-797.

Rademaker, R. L., Bloem, I. M., De Weerd, P., \& Sack, A. T. (2015). The impact of interference on short-term memory for visual orientation. Journal of Experimental Psychology: Human Perception and Performance, 41(6), 1650. 
Smith, E. E., Jonides, J., Koeppe, R. A., Awh, E., Schumacher, E. H., \& Minoshima, S. (1995). Spatial versus object working memory: PET investigations. Journal of Cognitive Neuroscience, 7(3), 337-356.

Van der Stigchel, S., Merten, H., Meeter, M., \& Theeuwes, J. (2007). The effects of a task-irrelevant visual event on spatial working memory. Psychonomic Bulletin \& Review, 14(6), 1066-1071.
White, J. M., Sparks, D. L., \& Stanford, T. R. (1994). Saccades to remembered target locations: An analysis of systematic and variable errors. Vision Research, 34(1), 79-92.

Zhang, W., \& Luck, S. J. (2009). Sudden death and gradual decay in visual working memory. Psychological Science, 20(4), 423-428. 